\title{
Mitochondrial DNA mutations, apoptosis, and the misfolded protein response.
}

Justin L. Mott

University of Nebraska Medical Center, justin.mott@unmc.edu

Dekui Zhang

Saint Louis University

Hans Peter Zassenhaus

Saint Louis University

Tell us how you used this information in this short survey.

Follow this and additional works at: https://digitalcommons.unmc.edu/com_bio_articles

Part of the Medical Biochemistry Commons, and the Medical Molecular Biology Commons

\section{Recommended Citation}

Mott, Justin L.; Zhang, Dekui; and Zassenhaus, Hans Peter, "Mitochondrial DNA mutations, apoptosis, and the misfolded protein response." (2005). Journal Articles: Biochemistry \& Molecular Biology. 9.

https://digitalcommons.unmc.edu/com_bio_articles/9

This Article is brought to you for free and open access by the Biochemistry \& Molecular Biology at DigitalCommons@UNMC. It has been accepted for inclusion in Journal Articles: Biochemistry \& Molecular Biology by an authorized administrator of DigitalCommons@UNMC. For more information, please contact digitalcommons@unmc.edu. 


\title{
Mitochondrial DNA Mutations, Apoptosis, and the Misfolded Protein Response
}

\author{
JUSTIN L. MOTT,* DEKUI ZHANG,* and HANS PETER ZASSENHAUS
}

\begin{abstract}
Studies of transgenic mice with accelerated accumulation of mtDNA mutations specifically in the heart lead us to propose that apoptotic signaling and cell death is central to the pathogenesis of mtDNA mutations in aging. It is the cellular response to that apoptotic signaling and the organ's compensatory response to a loss of cells that specify the phenotype of an accumulation of mtDNA mutations. In the heart, cardiomyocytes induce a vigorous anti-apoptotic, pro-survival response to counteract mitochondrial apoptotic signaling. The heart up-regulates contractility of remaining myocytes in order to maintain cardiac output. We hypothesize that mutant mitochondrial proteins originate apoptotic signaling by interacting with proteins already in place in the mitochondrial outer membrane that regulate apoptosis, for example the pro-apoptotic protein Bak. Since it is unlikely that all mutant mitochondrial proteins have the necessary structure and localization within the inner membrane to activate Bak appropriately, only a small fraction of an age-associated burden of mtDNA mutations may be pathogenic. In this model, reactive oxygen species generated by mitochondrial respiration drive the formation of mtDNA mutations, but are not the primary mechanism for their pathogenicity.
\end{abstract}

$\mathbf{M}$ ITOCHONDRIAL DNA (mtDNA) mutations accumulate with age and disease. ${ }^{1}$ These sporadic mutations randomly affect the mitochondrial genome, and thus a mutation at any particular nucleotide position is rare. Even with advanced age, the frequency of mutations generally does not exceed $1 \%$ in otherwise normal individuals. ${ }^{2}$ Thus, for these mutations to cause disease or senescence, the cell needs to be very sensitive to mtDNA mutations. Recent evidence suggests that the burden of point muta- tions in mtDNA may be higher than previously thought, ${ }^{3}$ so that estimates extrapolate to an average of three point mutations per genome in the aged brain. ${ }^{4}$ Still, given the random distribution of those mutations and the multiple copies of mtDNA per mitochondrion, their effect on respiratory function would be expected to be slight.

Despite their low frequency, mtDNA mutations are still more common than nuclear DNA mutations. Because of the compact nature of

*Each of these authors contributed equally to this manuscript.

Department of Molecular Microbiology and Immunology, St. Louis University Health Science Center, St. Louis, Missouri. 
the mitochondrial genome, these mutations are also more likely to affect a coding region. Due to heterogeneity, the mutation level may be higher in a given cell than the overall average of the tissue. ${ }^{5}$ At the next level of compartmentalization, the mitochondrion itself will have an even higher proportion of mutant genomes. Consider a cell that has only a single mutation on a background of maybe 1000 wildtype mitochondrial genomes. The tissue mutation level may be undetectable, the cellular mutation level would be $1 / 1000$ genomes. But the level of the mutation in the affected mitochondrion would be $10-50 \%$ (given two to 10 genomes in this prototypical mitochondrion). If mtDNA mutations contribute to disease, the signaling mechanism must sense the high proportion of mutation within a mitochondrion, not the low proportion of a cell or tissue.

The central tenet of the mitochondrial theory for aging is that those signals are reactive oxygen species (ROS), natural byproducts of mitochondrial respiration. ${ }^{6,7}$ The ensuing oxidative damage mutates mtDNA, leading in turn to even more ROS because of malfunctioning respiratory enzyme complexes, all of which, with the exception of Complex II, contain protein subunits encoded within mtDNA. ${ }^{8}$ The "vicious cycle" of ever increasing frequencies of mtDNA mutations and oxidative damage ultimately causes cellular senescence, in part because of accruing oxidative damage and in part because of declining mitochondrial oxidative phosphorylation. ${ }^{9}$

We argue here that apoptotic signaling is the primary pathogenic mechanism of mtDNA mutations in aging. It is now well established that mitochondria play a central role in amplifying cellular apoptotic signals within the intrinsic pathway for apoptosis. ${ }^{10}$ It is within mitochondrial membranes where interactions take place among pro- and anti-apoptotic proteins such as Bak, Bax, and $\mathrm{Bcl} 2$ to regulate outer membrane permeabilization and cytochrome $c$ release, unleashing the caspase cascade culminating in apoptosis. ${ }^{11}$ We propose that mutant mitochondrially encoded proteins tap into that machinery already in place so as to originate apoptotic signals. At any one moment, most cells sensing those signals suppress execution of apoptosis by up-regulation of anti- apoptotic proteins. Nevertheless, some cells succumb. Finally, we will present a hypothesis bearing on the molecular mechanism(s) for how mutant proteins might originate apoptotic signaling - a process we view as a mitochondrial misfolded protein response.

\section{LITERATURE REVIEW}

Transgenic mice with accelerated accumulation of mtDNA mutations

In order to fully develop this hypothesis, we will review recent results from a mouse model that accumulates mitochondrial DNA mutations at an accelerated rate. Transgenic mice were generated that accelerate accumulation of mtDNA mutations specifically in the heart because of the expression of a proof-reading deficient pol $\gamma$, the mtDNA polymerase. ${ }^{12}$ Since the $\alpha$-myosin heavy chain promoter driving expression of the transgene turns on only after birth, developmental abnormalities are avoided which might otherwise arise from fetal expression of the transgene. Likewise, since expression is restricted to cardiomyocytes, pathogenesis in the heart is not complicated by dysfunction in other organ systems, which could impact on cardiac function. This potentially confounding situation is of course inherent to characterizations of aged versus young animals or individuals. Similar interpretive difficulties are present with the recently reported knockin mouse where the wild-type pol $\gamma$ has been replaced by a proof-reading deficient version. ${ }^{13,14}$ In that model, mtDNA mutations are generated in all tissues, presumably from early embryogenesis onwards, it is more difficult to distinguish pathology arising directly from mtDNA mutations within a specific tissue from pathology secondary to homeostatic imbalances in the animal.

In our transgenic mice, mtDNA mutations rapidly accumulate specifically in the heart from birth onwards. By 1 month of age, point mutations reach levels of one per $10,000 \mathrm{bp}$ of mtDNA, determined by DNA sequencing of cloned fragments generated without PCR amplification. By 3 months of age, mutation frequencies climb to 1.5 per $10,000 \mathrm{bp}$, represent- 
ing on average two point mutations per mtDNA molecule. Mutations are randomly scattered around the mitochondrial genome and 5-10-fold higher than in wild-type animals. Humans and rodents also accumulate large scale deletion mutations in mtDNA with age. ${ }^{15,16}$ These transgenic mice do likewise. Thus, at the DNA level these mice recapitulate, albeit at an accelerated pace, features of mtDNA mutations found in aging humans and rodents.

Cardiac pathology is first observed in these transgenic mice at about 1 month of age. ${ }^{17}$ Transgenic animals invariably develop a fourchamber dilated cardiomyopathy, atrial thrombosis, fibrosis, and histologically apparent cell death. ${ }^{17}$ Conditions deteriorate over time so that animals rarely live beyond $1^{1 / 2}$ years of age, with terminal signs and symptoms consistent with end-stage heart failure. Even at younger ages (1-2 months) some animals develop congestive heart disease and experience sudden death.

The severe pathology observed in these mice seems to be inconsistent with the relatively benign frequencies of mtDNA mutations (i.e., $\sim 1$ mutation per $10,000 \mathrm{bp}$ ), raising concerns that artifacts associated with the transgene may be responsible for pathology, rather than the mutations themselves. Insertional mutagenesis of a specific nuclear gene by the transgene itself appears not to be involved since two independent lines show essentially identical phenotypes. Although transgene expression is some $2-4$-fold higher than endogenous pol $\gamma$ in wildtype animals, transgenic animals expressing a normal version of pol $\gamma$ at similar levels, driven by the same $\alpha$-MHC promoter, show no cardiac pathology and no accumulation of mtDNA mutations. ${ }^{17}$ DNA sequencing of the entire transgene used for construction of transgenic mice, including sequences recovered by PCR amplification from mouse chromosomal DNA, show no second site alterations (unpublished data). In the transgenic heart, mtDNA content does not differ from control littermates, indicating minimal effects of the mutant proofreading domain on the catalytic property of the enzyme. ${ }^{12}$ This is consistent with studies where we showed that expression of the mutant versus wild-type transgene in cos cells yielded
mtDNA polymerases with similar DNA synthetic activity, but as expected, the mutant enzyme lacked 3' exonuclease activity. ${ }^{12}$ Importantly, cardiac pathology in transgenic animals correlates with the rise in the frequency of mtDNA mutations rather than with expression of the transgene. By day 2 after birth, transgene expression is maximal, yet for the first 3-4 weeks of life cardiac structure appears to be completely normal at the gross and histological level. ${ }^{17}$

Finally, we now better appreciate that a frequency of point mutations of about two per genome may not be so benign. ${ }^{18}$ As pointed out in the Introduction, the aging human brain is estimated to have about three point mutations per genome, and this is considered a high burden. Similar to our mice, knockin mice expressing proof-reading deficient pol $\gamma$ in all tissues have point mutation frequencies in the heart about 4-6-fold higher than wild-type controls. ${ }^{13,14}$ These mice similarly develop a dilated cardiomyopathy. What these mouse models force us to face is that seemingly low frequencies of randomly generated mtDNA mutations are indeed pathogenic. They provide a strong counterweight to those arguing that the age-related rise in mtDNA mutations is an epiphenomenon, not being high enough to cause either disease or physiological senescence. ${ }^{19,20}$

\section{No decreased respiration or increased oxidative stress}

We first tested the hypothesis that pathology arose due to either decreased mitochondrial respiration or increased oxidative stress. We focused on animals 1-2 months of age, when pathology was first apparent so as to minimize potential complications secondary to longstanding disease. No evidence was found for either process. ${ }^{12,17,21}$ Specifically, with regards to respiration:

1. By polarography, isolated mitochondria from transgenic hearts showed no declines in respiration rates or $\mathrm{P}: \mathrm{O}$ ratios with a variety of substrates. ${ }^{12,17,21}$

2. Mitochondrial content of cytochromes was normal. ${ }^{12,17,21}$ 
3. Specific activities of the cytochrome oxidase, NADH-dehydrogenase, and ATP synthetase were normal ${ }^{17,21}$ (and unpublished data).

4. Substrate driven increases in the transmembrane potential of isolated mitochondria were normal. ${ }^{12,17,21}$

5. By western blotting, the content was normal for cytochrome $c$ and cox4, a mitochondrially encoded subunit of cytochrome oxidase. ${ }^{17}$

Furthermore, indirect evidence for a decline in respiratory function was lacking. The content of ATP was not lower in the transgenic heart; indeed, it was somewhat elevated. ${ }^{17}$ In patients with mtDNA mutations high enough to compromise oxidative phosphorylation, compensatory responses up-regulate both mitochondrial gene expression and number. ${ }^{22} \mathrm{By}$ Northern blotting $12,17,21$ and electron microscopy, ${ }^{17}$ neither of those compensatory responses was seen in transgenic hearts. Indeed, our collaborator performing the ultrastructural analysis of tissue sections from 6-week-old animals could not distinguish transgenic from littermate controls when blinded to their identity.

Specifically with regards to oxidative stress:

1. No increases were seen in the transgenic heart of either 8-OH-dG adducts in mtDNA or cellular and mitochondrial protein carbonyls, both indicators of oxidative damage. ${ }^{21}$

2. Glutathione levels and the ratio of GSH/ GSSG were unaltered. ${ }^{21}$

3. Total and specific activities of mitochondrial aconitase, an enzyme highly sensitive to oxidative damage, were normal. ${ }^{21}$

4. Upregulation of anti-oxidant defenses (e.g., copper/zinc superoxide dismutase, glutathione peroxidases) was not seen, either by immunohistochemistry ${ }^{21}$ or by microarray analysis. $^{23}$

5. Activation was not seen of signaling pathways responsive to oxidative stress (e.g., MAPK, SAPK/JNK, NF $\kappa$ B [unpublished data]).

6. Recent data from a separate model of increased mitochondrial DNA mutations in the whole-animal confirm that there is no increased oxidative stress. ${ }^{14}$
Collectively, the conclusion seems inescapable that elevated frequencies of mtDNA mutations in this model cause pathology by a mechanism not directly related to disturbances in the function of the electron transport chain.

\section{MtDNA mutations induce a pro-survival response at the cellular level}

At 1 month of age, when mice first show cardiac dilation, a surge of apoptosis occurs in the heart so that, at its peak, about one in 50 cardiomyocytes are TUNEL positive. ${ }^{17}$ Histologically, degenerating cells and cell drop-outs are visible by hematoxylin-eosin staining. Increased levels of cytosolic cytochrome $c$ indicate mitochondrial involvement in the apoptotic signaling. But apoptosis wanes over the ensuing weeks, so that by 8-10 weeks of age, frequencies of TUNEL-positive cells are only slightly higher than that of controls. ${ }^{17}$ Likewise, cytosolic levels of cytochrome $c$ decline but remain still slightly greater than controls. The decline in apoptosis correlates with a cellular pro-survival response, ${ }^{17,24,25}$ a coordinated upregulation of multiple anti-apoptotic proteins, including Bcl2, Bfl1, Bcl-XL, XIAP, and Hsp27, which function to suppress programmed cell death at various steps in the intrinsic pathway of apoptosis. ${ }^{26,27}$ This pro-survival response persists for the life of the animal, possibly because pro-apoptotic signaling persists, as evidenced by cytochrome $c$ release and mitochondrial associated Bax. Importantly, the pro-survival response occurs in all cardiomyocytes within the heart, as shown by immunohistochemistry for Bcl2 and Hsp27. ${ }^{17}$ It places the heart in a protective state against insults provoking cell death, ${ }^{25}$ such as doxorubicin, a cardiotoxin that causes apoptosis of cardiomyocytes. ${ }^{28}$

These results tell us that, at the cellular level, increased frequencies of mtDNA mutations provoke apoptotic signaling. Even at its peak, however, levels of cytosolic cytochrome $c$ in the transgenic heart are less than $5 \%$ of the total, suggesting that only a small fraction of mitochondria release cytochrome $c$ at any one time. This is consistent with cell culture studies indicating that cytochrome $c$ release is an all-ornone phenomenon at the mitochondrial level. ${ }^{29}$ The implication is that it may not be the cellu- 
lar burden of mtDNA mutations per se that is pathogenic, but rather either a threshold level in some mitochondria or a specific mutation in particular mitochondria. Since cardiomyocytes contain thousands of mitochondria, each harboring several mtDNA molecules, some mitochondrion somewhere in the cell is bound to harbor any specific mutation when the global average of random mutations climbs to $\sim 2$ per genome. The unfortunate few who have an apoptosis-signaling mutation, however, have a disproportionate effect on the entire population because of the cellular pro-survival response, which, for instance, places high levels of $\mathrm{Bcl} 2$ on all mitochondria. ${ }^{25}$

It is the pro-survival response that represents an important aspect of the pathogenesis of an age-associated rise in mtDNA mutations. Bcl2 in the endoplasmic and sarcoplasmic reticulum has been reported to alter permeability to calcium. ${ }^{30}$ Enforced over-expression of Bcl2 in the mouse heart inhibits the mitochondrial sodium-calcium exchanger and inhibits opening of the mitochondrial permeability transition pore. ${ }^{31}$ Higher levels of Bcl-XL, Bfl1, XIAP, Hsp27, and other participants in the pro-survival response would likewise be expected to affect cellular functions. Thus, the pro-survival response provoked by pro-apoptotic signaling from a few mitochondria is one mechanism for amplifying the pathogenic potential of a relatively low global average of mtDNA mutations in a cell. At the moment, we do not know what the molecular signaling pathways in cardiomyocytes are that up-regulate expression of $\mathrm{Bcl} 2$ and other genes involved in the pro-survival response.

\section{Cardiomyocytes in a pro-survival state have enhanced physiological function}

Is the pro-survival response acutely pathologic for cardiomyocytes? To address that question, we isolated single cells from dilated hearts 2-3 months of age and measured their contractility upon field stimulation at pacing frequencies of $0.5-4 \mathrm{~Hz}$ (Zhang et al., submitted). Surprisingly, transgenic cells have much greater contractility than control cells, showing nearly 1.8-fold greater fractional shortening than con- trols at all pacing frequencies. Increased storage of calcium in the sarcoplasmic reticulum and increased release of calcium during the transient after electrical stimulation appear to play an important role in that increased contractility. This hypercontractility indicates that transgenic cardiomyocytes are not physiologically dysfunctional or senescent, despite having higher levels of mtDNA mutations and despite being in a pro-survival state. These results seem paradoxical in that the heart is dilated and diseased, yet the cells therein are functioning at a supraphysiological level. The resolution to that paradox rests in the observation that, by 3 months of age, the transgenic heart has about $45 \%$ fewer cardiomyocytes than littermate controls (Zhang et al., submitted). Presumably, this is a consequence of the low levels of persistent apoptosis in the transgenic heart. Hypercontractility of the remaining cells, then, is a compensatory response of the heart to maintain cardiac output in the setting of a much reduced cell number. Whether that hypercontractility response is mechanistically related to or independent of the pro-survival response is unknown at the moment.

These results provide a new framework for viewing the pathogenesis of elevated levels of mtDNA mutations in the heart with age. As the burden of random mtDNA mutations rises, a few mitochondria within a cell happen to suffer the "right" mutation and initiate proapoptotic signaling. In cells with large numbers of mitochondria, such as cardiomyocytes, eventually all cells are affected. Although a vigorous anti-apoptotic, pro-survival response mostly suppresses execution of the apoptotic command, some cells succumb nevertheless. However, new mtDNA mutations continue to be generated with age, and the burden of mtDNA mutations inexorably climbs. Cells continue to be lost. The heart compensates for the lost cells by up-regulating the physiological function of the remaining cells. But eventually in a post-mitotic organ that compensation is limited, leading to either disease or physiological dysfunction at the organ level. The evoked pro-survival response in the absence of cell death may also by itself be maladaptive in the long term. This 
model, then, views apoptotic signaling as the primary pathogenic mechanism for rising levels of mtDNA mutations.

\section{Role of ROS}

In distinction to the free radical theory of aging, this model for the pathogenesis of an agerelated rise in mtDNA mutations does not directly implicate generation of ROS as the primary mechanism for their pathogenicity. However, oxidative damage still plays an important role. It is likely that in normal individuals oxidative damage is the main driving force for the generation of mtDNA mutations in the first place, resulting from the ROS generated by mitochondrial respiration. It would be interesting to know whether the pro-survival response itself, or other cellular compensatory responses that are invoked to maintain physiological function of the organ, have direct effects on ROS generation as well.

An apoptotic model for the pathogenesis of mtDNA mutations has implications for research using model systems to study their effects. If only rare, "correct" mtDNA mutations generate mutant proteins capable of interacting with the apoptotic machinery so as to signal apoptosis, then those pathogenic mutations would tend to be lost from a tissue as it ages and cells die. A powerful strategy to study the pathology of mtDNA mutations that are present in aged or diseased tissues is to fuse enucleated cells taken from such donor tissues with recipient cells in culture lacking mtDNA (i.e., $r h o^{0}$ cells). ${ }^{32}$ Even if the pro-survival response mostly suppresses apoptosis, allowing apoptosis-signaling mutations to persist longer than they would otherwise, cytoplasmic hybrids (cybrids) reconstituted with mitochondria from such tissues may not invoke a pro-survival response, either at all or fast enough. Again, by the time the cybrid cultures are established, the pathogenic mtDNA mutations might be lost. In an ironic twist, those who argue that age-associated mtDNA mutations are an epiphenomenon-a consequence of aging but not its cause-may be right. Not because mutagenesis of mtDNA is hardly high enough to be pathogenic, but because the pathogenic mutations are lost over time as cells die, leaving behind those mutations with little or reduced pathogenicity.

Mitochondrial permeability transition pore as a target for mtDNA mutations

This model then directs attention to how mtDNA mutations, or more accurately mutant mitochondrial proteins, originate apoptotic signaling (i.e., outer membrane permeabilization and release of cytochrome $c$ ). One such target may be the mitochondrial permeability transition pore (mPTP). This pore is a channel that spans the mitochondrial inner and outer membranes and is thought to be comprised of a complex of proteins including the voltage dependent anion channel located in the outer membrane and the adenine nucleotide translocator located in the inner membrane ${ }^{33}$ (for further complexities on the structure of the pore, see others $\left.{ }^{34,35}\right)$. Pore opening breaks down the osmotic barrier, which then leads to mitochondrial swelling, rupture of the outer membrane, and cytochrome $c$ release. ${ }^{36}$ Within the mitochondrial matrix, the activity of cyclophilin D (CyP D) facilitates pore opening. ${ }^{37}$ Cyp D is a chaperone having peptidyl-prolyl cis-trans isomerase activity and is the major cyclosporin A (CsA) binding protein within mitochondria. ${ }^{38}$ CsA is a potent inhibitor of pore opening. ${ }^{39}$ In rodent models for ischemic and traumatic brain injury and cardiac injury following ischemiareperfusion, CsA treatment protects against neuronal and myocytic cell death, ${ }^{40-42}$ suggesting that pore opening plays an important role in the apoptosis observed in those models. However, pore opening is not obligatory for cytochrome $c$ release and apoptosis in all circumstances. Cells in culture can release cytochrome $c$ and initiate apoptosis without pore opening, ${ }^{43}$ reflecting perhaps the intrinsic ability of pro-apoptotic proteins such as Bak and Bax to form channels within the outer membrane large enough for cytochrome $c$ to escape. ${ }^{11,44,45}$

In order to determine if the pore is a target for apoptotic signaling arising from mtDNA mutations, we treated our transgenic mice with 
CsA starting at 3 weeks of age (before disease is apparent) and continuing out to 6 weeks of age (when disease is well established). ${ }^{46} \mathrm{CsA}$ treatment completely prevented the development of cardiac dilation. Mutation-induced apoptosis was prevented, as was the induction of the pro-survival response, as indicated by the failure of $\mathrm{Bcl} 2$ to be up-regulated. Another aspect of the cardiomyopathy in these transgenic mice is profound down-regulation of the gap junction protein, connexin $43 .{ }^{46} \mathrm{CsA}$ treatment prevented that down-regulation as well. Control animals treated with FK506 (which, like CsA, inhibits cytoplasmic calcineurin activity, an important regulator of myocytic function, but unlike CsA does not inhibit mitochondrial Cyp D) showed none of the protective effects seen with CsA. ${ }^{46}$

These data implicate Cyp D function as necessary for the pathogenicity of elevated levels of mtDNA mutations. Is that function its facilitation of mPTP opening, implying that mutant mitochondrial proteins activate $\mathrm{CyP} \mathrm{D}$, so as to potentiate pore opening? In the transgenic heart, Cyp D is found associated with the mitochondrial inner membrane (unpublished data), a feature that is linked with potentiation of pore opening. ${ }^{47,48}$ Calcium overload is a potent trigger of pore opening, ${ }^{49}$ manifested in vitro as a concentration-dependent dissipation of the mitochondrial transmembrane potential. Transgenic mitochondria show resistance to pore opening (Mott et al., submitted). This resistance to pore opening is not what we would have expected if mutant mitochondrial proteins potentiate pore opening, similar to, for example, the effects of oxidant treatment on isolated mitochondria. 47,50

Further studies indicated that this resistance is a consequence of the pro-survival response. $\mathrm{Bcl} 2$ in mitochondrial membranes inhibits pore opening. ${ }^{31,51}$ In our transgenic animals, resistance to pore opening correlates in time with the up-regulation of $\mathrm{Bcl} 2$, which we know includes 5-10-fold higher levels of $\mathrm{Bcl} 2$ in mitochondrial membranes. ${ }^{25}$ Finally, pore opening in transgenic mitochondria is normalized by treatment with peptides whose sequences derive from the $\mathrm{BH} 3$ domains of $\mathrm{Bax}$ and $\mathrm{Bid}$ (Mott et al., in preparation). Bax and Bid bind $\mathrm{Bcl} 2$ through their $\mathrm{BH} 3$ domains, ${ }^{52}$ and pep- tides derived from those sequences interfere with the ability of $\mathrm{Bcl} 2$ to protect against pore opening and cytochrome $c$ release. ${ }^{53,54}$ These findings indicate that pore dysfunction in transgenic mitochondria is due to up-regulation of $\mathrm{Bcl} 2$ - and that pore dysfunction is one of the consequences of the pro-survival response. They suggest that potentiation of pore opening is not the mechanism driving apoptotic signaling by mutant mitochondrial proteins.

\section{HYPOTHESIS}

Molecular mechanism of apoptotic signaling by mtDNA mutations

If it isn't the pore, then what is the target for mutant mitochondrial proteins? CsA treatment of these animals strongly protects against development of disease and prevents apoptotic signaling. We suggest that it is inhibition of the chaperone function of CyP D by CsA, not inhibition of its pore-opening function, that abrogates apoptotic signaling of mutant mitochondrial proteins. All mitochondrial-encoded proteins are integral membrane subunits of the respiratory enzyme complexes, whose assembly and insertion into the inner membrane require the activity of chaperones. ${ }^{55} \mathrm{CyP} \mathrm{D}$ activity enhances the kinetics of assembly, ${ }^{56,57}$ although its activity seems not to be essential, most elegantly demonstrated by the recent reports of CyP D null mice in which mitochondrial function (aside from pore opening) appears to be quite normal. ${ }^{58-60}$ Thus, inhibition of CyP D chaperone activity by CsA would be expected to at least delay the translocation of newly made mutant mitochondrial proteins into the inner membrane. Since misfolded mutant proteins within mitochondria are degraded more rapidly than their wild-type counterparts, ${ }^{61-63}$ this delay by itself may be sufficient to substantially reduce the levels of mutant proteins inserted into the mitochondrial inner membrane.

If inserted into the inner membrane, mutant mitochondrial proteins would be poised to activate latent pro-apoptotic proteins. One such candidate is Bak, a resident integral outer membrane protein, whose conformational activation results in oligomerization, permeabilization of the outer membrane, release of cytochrome $c$, 
and apoptosis. ${ }^{64}$ Since it is unlikely that simply any mutant protein has the necessary structure to interact appropriately with Bak, stochastic mutagenesis of mtDNA would need to continue for some time so as to have a reasonable probability of generating the appropriate mutant. Recall that we earlier suggested that it may not be the burden of mutations per se that triggers apoptosis with aging, but rather specific rare mutants. Thus, apoptotic signaling may derive only from specific mutations in some mitochondrial genes, perhaps only those whose mutant protein product is situated within the inner membrane in such a way so as to be able to interact with Bak.

\section{Mitochondrial misfolded protein response}

We term this postulated mechanism for the pathogenicity of mutant mitochondrial proteins the mitochondrial misfolded protein response. The hypothesis that misfolded mitochondrial proteins originate apoptotic signaling has several attractive features. First, the level of misfolded proteins would be a sensitive indicator of stress. Remember the example given in the Introduction of a cell with one mutation in a 1000; a respiratory decrease would be all but undetectable. By detecting a positive signal over a low background (misfolded proteins), the misfolded response would be sensitive to this low level of damage. Amplification of its impact on cellular function would follow from the pro-survival response. Further, the offending mutation would not be ignored through the complementing gene product of another mitochondrial genome in the same organelle. Another feature of this response system is that the insult need not necessarily derive from mtDNA mutations. Other stresses can cause misfolding, including heat stress and oxidative damage. In the case of oxidative stress, it would be efficient to have a detector of ROS in close proximity to a major generator of ROS, the electron transport chain. Finally, the sensitivity of the cellular response to misfolded proteins could be fine tuned to the demands of the cell via regulation by protective proteins like $\mathrm{Bcl} 2$. So, for example, the maternal germ cell would be a cell where a low signal from mitochondria activates cell death, con- tributing to the high rates of atresia and apoptosis during oocyte development. ${ }^{65}$ Having high sensitivity would help ensure that newborns are remarkably free of mutations. ${ }^{66}$ There are likely additional safeguards in development that are beyond the scope of this review, including a mitochondrial genetic bottleneck. ${ }^{67}$ Still, some mutations would bypass detection by the mitochondrial misfolded protein response, as they would not result in misfolded proteins (e.g., deletion mutations which completely inactivate mitochondrial translation). Some potential examples of mutations that bypass selection are inherited mitochondrial myopathies ${ }^{68}$ and common polymorphisms. ${ }^{69}$

\section{Testing the hypothesis}

This apoptotic model for the pathogenesis of mtDNA mutations makes some testable predictions:

1. Pathology in mice expressing a proof-reading deficient pol $\gamma$ should be ameliorated when the transgene is placed on a background null for CyP D or Bak. Null mice for both of those proteins are available. ${ }^{59,70} \mathrm{In}$ deed, CyP D and Bak null mice, which by themselves show remarkably little pathology, might show increased longevity since the apoptotic pathogenicity of mtDNA mutations has been blunted.

2. Cybrids reconstituted with mitochondria from aged tissues might reveal the apoptotic potential of mtDNA mutations in the tissue if the cybrid recipients over-express Bcl2 under control of a regulatable promoter. Once established under high $\mathrm{Bcl} 2$ expression, down-regulating $\mathrm{Bcl} 2$ in these cultures may trigger a wave of apoptosis. The underlying assumption here is that cells from aging tissues retain mitochondria with apoptotic signaling mutations because of up-regulation of a pro-survival response.

3. Placing transgenic mice with rapid accumulation of mtDNA mutations on a Bcl2-deficient background should greatly increase the pathogenicity of mtDNA mutations. Preliminary results with our transgenic mice indicate that this is indeed the case.

4. Finally, centenarians might exhibit poly- 
morphisms in the genes for CyP D or Bak that result in reduced transduction of the pathogenicity of mtDNA mutations.

\section{CONCLUSION}

It needs to be remembered that aging is a complex, multifactorial process. This apoptotic signaling hypothesis addresses the pathogenicity of only point mutations in mitochondrial protein genes. We know that point mutations in tRNA genes and massive deletion mutations also accumulate with age, which have the potential to completely prevent translation in affected mitochondria. Their pathogenicity would be expected to involve different mechanisms. In aging tissues, cells with a heterogeneous spectrum of mutational types and amounts are likely to be present resulting in a complex interplay of pathologies. Our hypothesis hopefully will focus attention on apoptotic signaling so as to better determine its role in driving the aging process.

\section{ACKNOWLEDGMENTS}

We thank Drs. Clifford Bellone and Harris Perlman for helpful comments. This work was supported by grants from the National Institutes of Aging, and Neurological Disorders and Stroke, and the American Heart Association.

\section{REFERENCES}

1. Wallace DC, Shoffner JM, Trounce I, Brown MD, Ballinger SW, Corral-Debrinski M, Horton T, Jun AS, Lott MT. Mitochondrial DNA mutations in human degenerative diseases and aging. Biochim Biophys Acta 1995;1271:141-151.

2. Liu VW, Zhang C, Nagley P. Mutations in mitochondrial DNA accumulate differentially in three different human tissues during ageing. Nucleic Acids Res 1998;26:1268-1275.

3. Lin MT, Simon DK, Ahn CH, Kim LM, Beal MF. High aggregate burden of somatic mtDNA point mutations in aging and Alzheimer's disease brain. Hum Mol Genet 2002;11:133-145.

4. Smigrodzki R, Parks J, Parker WD. High frequency of mitochondrial complex I mutations in Parkinson's disease and aging. Neurobiol Aging 2004;25:1273-1281.
5. Khrapko K, Bodyak N, Thilly WG, van Orsouw NJ, Zhang X, Coller HA, Perls TT, Upton M, Vijg J, Wei JY. Cell-by-cell scanning of whole mitochondrial genomes in aged human heart reveals a significant fraction of myocytes with clonally expanded deletions. Nucleic Acids Res 1999;27:2434-2441.

6. Harman D. Aging: a theory based on free radical and radiation chemistry. J Gerontol 1956;11:298-300.

7. Harman D. The biologic clock: the mitochondria? J Am Geriatr Soc 1972;20:145-147.

8. Fleming JE, Miquel J, Cottrell SF, Yengoyan LS, Economos AC. Is cell aging caused by respiration-dependent injury to the mitochondrial genome? Gerontology 1982;28:44-53.

9. Linnane AW, Marzuki S, Ozawa T, Tanaka M. Mitochondrial DNA mutations as an important contributor to ageing and degenerative diseases. Lancet 1989;1:642-645.

10. Jiang X,Wang X. Cytochrome C-mediated apoptosis. Annu Rev Biochem 2004;73:87-106.

11. Korsmeyer SJ, Wei MC, Saito M, Weiler S, Oh KJ, Schlesinger PH. Pro-apoptotic cascade activates BID, which oligomerizes BAK or BAX into pores that result in the release of cytochrome c. Cell Death Differentiation 2000;7:1166-1173.

12. Zhang D, Mott JL, Chang SW, Denniger G, Feng Z, Zassenhaus HP. Construction of transgenic mice with tissue-specific acceleration of mitochondrial DNA mutagenesis. Genomics 2000;69:151-161.

13. Trifunovic A, Wredenberg A, Falkenberg M, Spelbrink JN, Rovio AT, Bruder CE, Bohlooly Y, Gidlof S, Oldfors A, Wibom R, Tornell J, Jacobs HT, Larsson NG. Premature ageing in mice expressing defective mitochondrial DNA polymerase. Nature 2004;429: 417-423.

14. Kujoth GC, Hiona A, Pugh WL, Someya S, Panzer K, Wohlgemuth SE, Hofer T, Seo AY, Sullivan R, Jobling WA, Morrow JD, Van Remmen H, Sedivy JM, Yamasoba T, Weindruch R, Leeuwenburgh C, Prolla TA. Mitochondrial DNA mutations, oxidative stress, and apoptosis in mammalian aging. Science 2005;309: 481-484.

15. Arnheim N, Cortopassi G. Deleterious mitochondrial DNA mutations accumulate in aging human tissues. Mutat Res 1992;275:157-167.

16. Wang E, Wong A, Cortopassi G. The rate of mitochondrial mutagenesis is faster in mice than humans. Mutat Res 1997;377:157-166.

17. Zhang D, Mott JL, Farrar P, Ryerse JS, Chang SW, Stevens M, Denniger G, Zassenhaus HP. Mitochondrial DNA mutations activate the mitochondrial apoptotic pathway and cause dilated cardiomyopathy. Cardiovasc Res 2003;57:147-157.

18. Smigrodzki RM, Khan SM. Mitochondrial microheteroplasmy and a theory of aging and age-related disease. Rejuvination Res 2005;8:172-198.

19. Rasmussen UF, Krustrup P, Kjaer M, Rasmussen HN. Experimental evidence against the mitochondrial theory of aging. A study of isolated human skeletal muscle mitochondria. Exp Gerontol 2003;38:877-886. 
20. Jacobs HT. The mitochondrial theory of aging: dead or alive? Aging Cell 2003;2:11-17.

21. Mott JL, Zhang D, Stevens M, Chang S, Denniger G, Zassenhaus HP. Oxidative stress is not an obligate mediator of disease provoked by mitochondrial DNA mutations. Mutat Res 2001;474:35-45.

22. Heddi A, Stepien G, Benke PJ, Wallace DC. Coordinate induction of energy gene expression in tissues of mitochondrial disease patients. J Biol Chem 1999;274: 22968-22976.

23. Zhang D, Ezekiel UR, Chang SW, Zassenhaus HP. Gene expression profile in dilated cardiomyopathy caused by elevated frequencies of mitochondrial DNA mutations in the mouse heart. Cardiovasc Pathol 2005;14:61-69.

24. Mott JL, Zhang D, Stevens M, Chang S, Denniger G, Zassenhaus HP. Oxidative stress is not an obligate mediator of disease provoked by mitochondrial DNA mutations. Mutat Res 2001;474:35-45.

25. Zhang D, Mott JL, Chang SW, Stevens M, Mikolajczak P, Zassenhaus HP. Mitochondrial DNA mutations activate programmed cell survival in the mouse heart. Am J Physiol 2005;288:H2476-H2483.

26. Danial NN, Korsmeyer SJ. Cell death: critical control points. Cell 2004;116:205-219.

27. Concannon CG, Gorman AM, Samali A. On the role of Hsp27 in regulating apoptosis. Apoptosis 2003;8: 61-70.

28. Zhang J, Clark JR Jr, Herman EH, Ferrans VJ. Doxorubicin-induced apoptosis in spontaneously hypertensive rats: differential effects in heart, kidney and intestine, and inhibition by ICRF-187. J Mol Cell Cardiol 1996;28:1931-1943.

29. Goldstein JC, Waterhouse NJ, Juin P, Evan GI, Green DR. The coordinate release of cytochrome c during apoptosis is rapid, complete and kinetically invariant Nat Cell Biol 2000;2:156-162.

30. Oakes SA, Opferman JT, Pozzan T, Korsmeyer SJ, Scorrano L. Regulation of endoplasmic reticulum $\mathrm{Ca}^{2+}$ dynamics by proapoptotic Bcl-2 family members. Biochem Pharmacol 2003;66:1335-1340.

31. Zhu L, Yu Y, Chua BH, Ho YS, Kuo TH. Regulation of sodium-calcium exchange and mitochondrial energetics by Bcl-2 in the heart of transgenic mice. J Mol Cell Cardiol 2001;33:2135-2144.

32. King MP, Attardi G. Human cells lacking mtDNA: repopulation with exogenous mitochondria by complementation. Science 1989;246:500-503.

33. Beutner G, Ruck A, Riede B, Welte W, Brdiczka D. Complexes between kinases, mitochondrial porin and adenylate translocator in rat brain resemble the permeability transition pore. FEBS Lett 1996;396:189-195.

34. Kokoszka JE, Waymire KG, Levy SE, Sligh JE, Cai J, Jones DP, MacGregor GR, Wallace DC. The ADP / ATP translocator is not essential for the mitochondrial permeability transition pore. Nature 2004;427:461-465.

35. He L, Lemasters JJ. Regulated and unregulated mitochondrial permeability transition pores: a new paradigm of pore structure and function? FEBS Lett 2002;512:1-7.
36. Zamzami N, Kroemer G. The mitochondrion in apoptosis: how Pandora's box opens. Nat Rev Mol Cell Biol 2001;2:67-71.

37. Crompton M. The mitochondrial permeability transition pore and its role in cell death. Biochem J 1999; 341:233-249.

38. Andreeva L, Tanveer A, Crompton M. Evidence for the involvement of a membrane-associated cyclosporin-A-binding protein in the $\mathrm{Ca}^{2+}$-activated inner membrane pore of heart mitochondria. Eur J Biochem 1995;230:1125-1132.

39. Halestrap AP, Davidson AM. Inhibition of $\mathrm{Ca}^{2+}$-induced large-amplitude swelling of liver and heart mitochondria by cyclosporin is probably caused by the inhibitor binding to mitochondrial-matrix peptidylprolyl cis-trans isomerase and preventing it interacting with the adenine nucleotide translocase. Biochem J 1990;268:153-160.

40. Friberg H, Ferrand-Drake M, Bengtsson F, Halestrap AP, Wieloch T. Cyclosporin A, but not FK 506, protects mitochondria and neurons against hypoglycemic damage and implicates the mitochondrial permeability transition in cell death. J Neurosci 1998; 18:5151-5159.

41. Okonkwo DO, Povlishock JT. An intrathecal bolus of cyclosporin A before injury preserves mitochondrial integrity and attenuates axonal disruption in traumatic brain injury. J Cereb Blood Flow Metab 1999; 19:443-451.

42. Griffiths EJ, Halestrap AP. Protection by Cyclosporin A of ischemia/reperfusion-induced damage in isolated rat hearts. J Mol Cell Cardiol 1993;25:1461-1469.

43. Goldstein JC, Munoz-Pinedo C, Ricci J-E, Adams SR, Kelekar A, Schuler M, Tsien RY, Green DR. Cytochrome $\mathrm{c}$ is released in a single step during apoptosis. Cell Death Differentiation 2005;12:453-462.

44. Antonsson B, Montessuit S, Lauper S, Eskes R, Martinou JC. Bax oligomerization is required for channelforming activity in liposomes and to trigger cytochrome c release from mitochondria. Biochem J 2000;345:271-278.

45. Kuwana T, Mackey MR, Perkins G, Ellisman MH, Latterich M, Schneiter R, Green DR, Newmeyer DD. Bid, Bax, and lipids cooperate to form supramolecular openings in the outer mitochondrial membrane. Cell 2002;111:331-342.

46. Mott JL, Zhang D, Freeman JC, Mikolajczak P, Chang SW, Zassenhaus HP. Cardiac disease due to random mitochondrial DNA mutations is prevented by cyclosporin A. Biochem Biophys Res Commun 2004; 319:1210-1215.

47. Connern CP, Halestrap AP. Recruitment of mitochondrial cyclophilin to the mitochondrial inner membrane under conditions of oxidative stress that enhance the opening of a calcium-sensitive non-specific channel. Biochem J 1994;302:321-324.

48. Connern CP, Halestrap AP. Chaotropic agents and increased matrix volume enhance binding of mitochondrial cyclophilin to the inner mitochondrial membrane and sensitize the mitochondrial perme- 
ability transition to $\left[\mathrm{Ca}^{2+}\right]$. Biochemistry 1996;35: 8172-8180.

49. Bernardi P. Mitochondrial transport of cations: channels, exchangers, and permeability transition. Physiol Rev 1999;79:1127-1155.

50. Tanveer A, Virji S, Andreeva L, Totty NF, Hsuan JJ, Ward JM, Crompton M. Involvement of cyclophilin $\mathrm{D}$ in the activation of a mitochondrial pore by $\mathrm{Ca}^{2+}$ and oxidant stress. Eur J Biochem 1996;238:166-172.

51. Zamzami N, Susin SA, Marchetti P, Hirsch T, GomezMonterrey I, Castedo M, Kroemer G. Mitochondrial control of nuclear apoptosis. J Exp Med 1996;183: 1533-1544.

52. Sattler M, Liang H, Nettesheim D, Meadows RP, Harlan JE, Eberstadt M, Yoon HS, Shuker SB, Chang BS, Minn AJ, Thompson CB, Fesik SW. Structure of BclxL-Bak peptide complex: recognition between regulators of apoptosis. Science 1997;275:983-986.

53. Vieira HL, Boya P, Cohen I, El Hamel C, Haouzi D, Druillenec S, Belzacq AS, Brenner C, Roques B, Kroemer G. Cell-permeable BH3-peptides overcome the cytoprotective effect of Bcl-2 and Bcl-X(L). Oncogene 2002;21:1963-1977.

54. Narita M, Shimizu S, Ito T, Chittenden T, Lutz RJ, Matsuda H, Tsujimoto Y. Bax interacts with the permeability transition pore to induce permeability transition and cytochrome c release in isolated mitochondria. Proc Natl Acad Sci USA 1998;95:14681-14686.

55. Kang PJ, Ostermann J, Shilling J, Neupert W, Craig EA, Pfanner N. Requirement for hsp70 in the mitochondrial matrix for translocation and folding of precursor proteins. Nature 1990;348:137-143.

56. Rassow J, Mohrs K, Koidl S, Barthelmess IB, Pfanner N, Tropschug M. Cyclophilin 20 is involved in mitochondrial protein folding in cooperation with molecular chaperones Hsp70 and Hsp60. Mol Cell Biol 1995;15:2654-2662.

57. Matouschek A, Rospert S, Schmid K, Glick BS, Schatz G. Cyclophilin catalyzes protein folding in yeast mitochondria. Proc Natl Acad Sci USA 1995;92:63196323.

58. Basso E, Fante L, Fowlkes J, Petronilli V, Fortes MA, Bernardi P. Properties of the permeability transition pore in mitochondria devoid of cyclophilin D. J Biol Chem 2005;280:18558-18561.

59. Baines CP, Kaiser RA, Purcell NH, Blair NS, Osinska $\mathrm{H}$, Hambleton MA, Brunskill EW, Sayen MR, Gottlieb RA, Dorn GW, Robbins J, Molkentin JD. Loss of cyclophilin D reveals a critical role for mitochondrial permeability transition in cell death. Nature 2005;434: 658-662.

60. Nakagawa T, Shimizu S, Watanabe T, Yamaguchi O, Otsu K, Yamagata H, Inohara H, Kubo T, Tsujimoto Y. Cyclophilin D-dependent mitochondrial permeability transition regulates some necrotic but not apoptotic cell death. Nature 2005;434:652-658.

61. de Jong L, Elzinga SD, McCammon MT, Grivell LA, van der SH. Increased synthesis and decreased sta- bility of mitochondrial translation products in yeast as a result of loss of mitochondrial $\left(\mathrm{NAD}^{+}\right)$-dependent isocitrate dehydrogenase. FEBS Lett 2000;483: 62-66.

62. Kogo T, Satoh Y, Kanazawa M, Yamamoto S, Takayanagi M, Ohtake A, Mori M, Niimi H. Expression analysis of two mutant human ornithine transcarbamylases in COS-7 cells. J Human Genet 1998; 43:54-58.

63. Xiao Q, Weiner H, Crabb DW. The mutation in the mitochondrial aldehyde dehydrogenase (ALDH2) gene responsible for alcohol-induced flushing increases turnover of the enzyme tetramers in a dominant fashion. J Clin Invest 1996;98:2027-2032.

64. Wei MC, Lindsten T, Mootha VK, Weiler S, Gross A, Ashiya M, Thompson CB, Korsmeyer SJ. tBID, a membrane-targeted death ligand, oligomerizes BAK to release cytochrome c. Genes Dev 2000;14:2060-2071.

65. Morita Y,Tilly JL. Oocyte apoptosis: like sand through an hourglass. Dev Biol 1999;213:1-17.

66. Munscher C, Rieger T, Muller-Hocker J, Kadenbach B. The point mutation of mitochondrial DNA characteristic for MERRF disease is found also in healthy people of different ages. FEBS Lett 1993;317:27-30.

67. Jansen RP, de Boer K. The bottleneck: mitochondrial imperatives in oogenesis and ovarian follicular fate. Mol Cell Endocrinol 1998;145:81-88.

68. Singh G, Lott MT, Wallace DC. A mitochondrial DNA mutation as a cause of Leber's hereditary optic neuropathy. N Engl J Med 1989;320:1300-1305.

69. Kogelnik AM, Lott MT, Brown MD, Navathe SB, Wallace DC. MITOMAP: a human mitochondrial genome database-1998 update. Nucleic Acids Res 1998;26: 112-115.

70. Lindsten T, Ross AJ, King A, Zong WX, Rathmell JC, Shiels HA, Ulrich E, Waymire KG, Mahar P, Frauwirth K, Chen Y, Wei M, Eng VM, Adelman DM, Simon MC, Ma A, Golden JA, Evan G, Korsmeyer SJ, MacGregor GR, Thompson CB. The combined functions of proapoptotic Bcl-2 family members bak and bax are essential for normal development of multiple tissues. Mol Cell 2000;6:1389-1399.

Address reprint requests to: Hans Peter Zassenhaus, Ph.D. Department of Molecular Microbiology and Immunology

St. Louis University Health Science Center 1402 S. Grand Blvd. St. Louis, MO 63104

E-mail: zassenp@slu.edu

Received: June 8, 2005 Accepted: August 10, 2005 
This article has been cited by:

1. A. Safdar, J. M. Bourgeois, D. I. Ogborn, J. P. Little, B. P. Hettinga, M. Akhtar, J. E. Thompson, S. Melov, N. J. Mocellin, G. C. Kujoth, T. A. Prolla, M. A. Tarnopolsky. 2011. Endurance exercise rescues progeroid aging and induces systemic mitochondrial rejuvenation in mtDNA mutator mice. Proceedings of the National Academy of Sciences 108:10, 4135-4140. [CrossRef]

2. Steven J. Dubec, Rajeev Aurora, H. Peter Zassenhaus. 2008. Mitochondrial DNA Mutations May Contribute to Aging Via Cell Death Caused by Peptides that Induce Cytochrome c Release. Rejuvenation Research 11:3, 611-619. [Abstract] [Full Text PDF] [Full Text PDF with Links] [Supplemental Material]

3. Tadafumi Kato. 2007. Mitochondrial Dysfunction as the Molecular Basis of Bipolar Disorder. CNS Drugs 21:1, 1-11. [CrossRef]

4. Aubrey D.N.J. de Grey. 2006. Rejuvenation Research in 2005. Rejuvenation Research 9:3, 413-415. [Citation] [Full Text PDF] [Full Text PDF with Links]

5. Konstantin Khrapko, Yevgenya Kraytsberg, Aubrey DNJ de Grey, Jan Vijg, Eric A. Schon. 2006. Does premature aging of the mtDNA mutator mouse prove that mtDNA mutations are involved in natural aging?. Aging Cell, ahead of print060426030539003. [CrossRef] 